\title{
Compressive spectrum sensing using two-stage scheme for cognitive radio networks
}

\author{
Montadar Abas Taher', Mohammad Z. Ahmed ${ }^{2}$, Emad Hmood Salman ${ }^{3}$ \\ ${ }^{1,3}$ Department of Communications Engineering, College of Engineering, University of Diyala, Iraq \\ ${ }^{2}$ Department of Communication and Electrical Engineering, Faculty of Engineering, \\ University of Al-Hussein Bin Talal, Jordan
}

\begin{tabular}{l} 
Article Info \\
\hline Article history: \\
Received Nov 1, 2019 \\
Revised May 14, 2020 \\
Accepted May 27, 2020 \\
\hline
\end{tabular}

Keywords:

Cognitive radio networks Compressive spectrum sensing

Discrete cosine transform Discrete wavelet transform Signal-to-noise ratio wall

\begin{abstract}
The modern applications of communications that use wideband signals suffer the lacking since the resources of this kind of signals are limited especially for fifth generation (5G). The compressive spectrum sensing (COMPSS) techniques address such issues to reuse the detected signals in the networks and applications of 5G. However, the raw techniques of COMPSS have low compression ratio and high computational complexity rather than high level of noise variance. In this paper, a hybrid COMPSS scheme has been developed for both non-cooperative and cooperative cognitive radio networks. The proposed scheme compiles on discrete wavelet transform single resolution (DWT-SR) cascaded with discrete cosine transform (DCT). The first is constructed according to the pyramid algorithm to achieve $50 \%$ while the second performed $30 \%$ compression ratios. The simulation and analytic results reveal the significant detection performance of the proposed technique is better than that of the raw COMPSS techniques.
\end{abstract}

Copyright (c) 2020 Institute of Advanced Engineering and Science. All rights reserved.

\section{Corresponding Author:}

Emad Hmood Salman,

Department of Communications Engineering,

College of Engineering, University of Diyala,

Ba'aqubah 32001, Diyala, Iraq

Email: emad.h.salman@ieee.org

\section{INTRODUCTION}

In the last two decades, the cognitive radio (CR) concept has attracted a lot of interest ever since it was used for efficiently utilizing spectrum, enabling coexistence for different wireless networks, weakening harmful interference, and increasing reliable wireless services [1, 2]. In the cognitive radio networks (CRNs), the secondary users (SUs) observe the spectrum bands and repeatedly detect the unoccupied ones using one of the spectrum sensing (SS) techniques. Thus, the chance of detecting spectrum increases relative to the increased width of the bandwidth [3,4]. To address these issues, the compressive spectrum sensing (COMPSS) technique is seen as a practicable key. Its fundamental is sampling the received primary user (PU) signal at rates that are below the Nyquist rate with conserving PU signal specifications to recover it accuratly $[5,6]$.

One of COMPSS technique issues is reducing the consumed power in the receivers via increasing the compression ratio rather than minimizing the high cost of Analog-to-Digital Converters (ADCs) design. Many of COMPSS techniques have been developed in literatures. In a recent study by Astaiza, et al., (2016), the COMPSS for cooperative users were performed using signal matrix estimation algorithm [7]. It was used for a multi-antenna case, but its detection performance was poor for low SNR. Wang [8] derived other algorithm for COMPSS according to positioning technology. It had low complexity since no reconstruction was needed but its performance was poor in low SNR. The discrete sine transform (DST) was also exploited for COMPSS with cooperative users [9]. Its detection performance was tested using the OR and Majority 
rules and showed a bad result at $\mathrm{SNR}<-5 \mathrm{~dB}$. In El-Khamy, et al., [3], the stationary wavelet transform (SWT) was studied and its performance was compared with discrete wavelet transform (DWT). However, its performance was defective for low SNR. The last but not least, the maximum inner product algorithm was derived [10]. This algorithm has a big compression ratio and an acceptable level of complexity, but it has a bad detection performance when $\mathrm{SNR}<-5 \mathrm{~dB}$.

The abovementioned disadvantages of the proposed COMPSS techniques are addressed in a higher compression ratio by proposed hybrid scheme. In addition, to reduce the consumed energy in ADC. Thus, the COMPSS technique involves decreasing the sampling rate lower than the Nyquist rate. This paper develops a hybrid COMPSS scheme in details by proposing a constant local and global false alarm ratios (CLFAR) and (CGFAR) expressions, and their effects on detection performance, compression ratio, SNR wall, and computational complexity trade-offs in sensing the OFDM signal [11-14]. The rest of this paper is outlined as follows: The algorithm of the considered COMPSS scheme and its formulated problem are described in section 2. In section 3, the proposed COMPSS technique scenarios are clarified and analyzed. Numerical and graphical results and their analyses are given in section 4. Conclusions of this paper are depicted in section 5 .

\section{THE BACKGROUND MODEL OF COMPSS TECHNIQUE}

The COMPSS technique show how to sample the signal less than the Nyquist rate to reduce the ADC cost while the raw approaches were sampled at the Nyquist rate. The COMPSS algorithm can often be performed via three series steps, as depicted in Figure 1 [15, 16]: spread description, sub-Nyquist sampling, and reconstruction. The functions of these steps are: (1) to spread the signal over reconstructable bases, (2) to sample the spreading signal at a sub-Nyquist ratio, (3) to reconstruct the sampling signal [17].

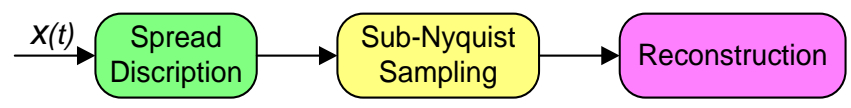

Figure 1. Block diagram of COMPSS steps

From the viewpoint of spectrum sensing (SS), the sensing detectors sense the various signals that have lower power compared to the wideband signals. However, there are some drawbacks such as using a number of RF frontends as the number of bands that should be sensed, computational complexity, and high consumption of energy, which results in air pollution. The COMPSS concept is more or less recovers the sensed signal that is sampled at the sub-Nyquist rate to meet the ADC demands and deal with issues of wideband signals sensing [18].

To describe the mathematical model of COMPSS, suppose that New is a spread samples number with $N e w \ll N$, and $N$ denotes the original length signal. In addition, suppose that $\psi$ is a spread bases (matrix) such as DFT or DWT with size of $(N \times N)$. Thus, the spread signal can be modelled as follows:

$$
x_{\text {COMP }}=\psi \times s
$$

where $x_{\text {СOMP }}$ denotes the spread signal and $s$ is the extension of signal where $\|s\|_{0}=N e w \ll N$. Suppose that $\boldsymbol{\Phi}$ is a sensing matrix with size of $(N e w \times N)$ to compress the signal with essential information of $x_{C O M P}$, as depicted in Figure 2.

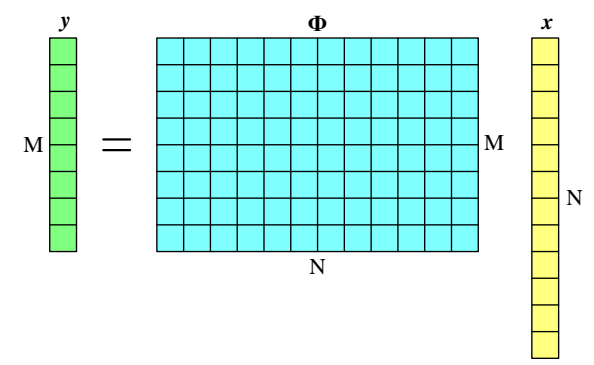

Figure 2. Structure of compression process 
The compressed signal, $y_{\text {СОMP }}$, represents the signal measurements of signal with New samples and can be modelled as:

$$
y_{C O M P}=\Phi \times x_{C O M P}
$$

To recover the original signal, the next equation will show how it can be achieved. From the small number $(\mathrm{New})$, the big number $(N)$ can be predicted due to the spreading assumption. This equation is considered an optimised problem to address this problem, as follows:

$$
\tilde{x}_{\text {СOMP }}=\arg \min _{y_{\text {COMP }}=\Phi \times x_{\text {COMP }}}\left\|x_{\text {COMP }}\right\|_{1} \quad \text { Subject to } \quad y_{\text {СOMP }}=\Phi \times x_{\text {СOMP }}
$$

where $\tilde{x}_{\text {СOMP }}$ is the reconstructed signal and $\left\|x_{\text {COMP }}\right\|_{m}=\sqrt[m]{\sum_{i}\left|x_{\text {COMP }}\right|^{m}}$ is the $\mathcal{L}_{m}$ norm of $x_{\text {COMP }}$ [17].

\section{THE PROPOSED HYBRID COMPSS SCHEME}

Basically, the COMPSS is applied to capture useful data (in time or frequency domains), so these data are compressed to counter the shortcomings of the bandwidth. Thus, it should be compressed by bases with superior resolution that keep the necessary measurements in order to reduce the cost. The proposed COMPSS system has been achieved through two cascaded stages: DDWT followed by CDCT for one SU (non-cooperative system) and DDWT followed by MCDCT for multi SUs (cooperative system), as shown in Figures 3 and 4, respectively.

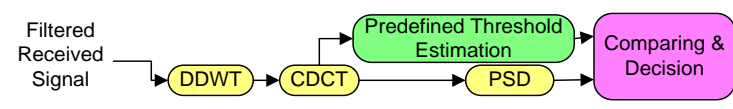

Figure 3. Hybrid COMPSS for one SU block diagram

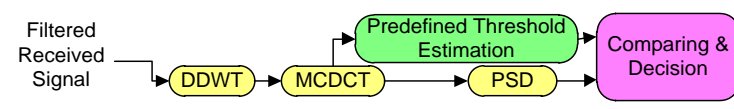

Figure 4. Hybrid COMPSS for multi SUs block diagram

The first stage essentially depends on WT, since it can analyse the signal to coefficients as their frequencies. For instance, the first decomposition level can categorise the signal into two components: low frequency that contains the traffic, and high frequency that contains the noise [19]. The Daubechies wavelets type is a higher base wavelet order rather than it is orthogonal. It is smoother and has better frequency localisation than others [20]. The Daubechies bases were extracted using the pyramid algorithm, which was investigated by Hansen, [21] and others. The pyramid algorithm generates the bases according to the required order number from the bases $d_{1}, d_{2}, d_{3}$, and $d_{4}$. Next, the new bases were created from averaging (as $d_{1}+d_{2} / 2$ ), and the new base is then moved up to a new level towards the top of the pyramid. From differencing (as $d_{1}-d_{2} / 2$ ), the new base is moved down to a new level towards the bottom of the pyramid, and so on in order to build the pyramid [21].

To build the DDWT matrix, $\boldsymbol{\Phi}_{1}$, as stated in the pyramid algorithm, the number of bases is required to be the same as the number of equations in order to obtain their rates. The basis vectors of the proposed matrix should be perpendicular, i.e., the result of the inner product between every two vectors equals to zero. The main four bases rates were obtained in [22] by I. Daubechies, as follows; $d_{1}=(1+\sqrt{3}) / 4, d_{2}=(3+\sqrt{3}) / 4$, $d_{3}=(3-\sqrt{3}) / 4$ and $d_{4}=(1-\sqrt{3}) / 4$. To complete the DDWT matrix for $1024 \times 1024$ vectors, the other bases can be constructed according to the pyramid algorithm. The scaling function and the three wavelet coefficients can be formulated as follows:

$$
\begin{aligned}
& s_{1}=\left(\sum_{a=1}^{\frac{N}{4}} d_{a}+2 \sum_{a=\left(\frac{N}{4}\right)+1}^{\frac{3 N}{4}} d_{a}+\sum_{a=(3 N / 4)+1}^{N} d_{a}\right) /(5 N / 2) \\
& w_{1}=\left(2 \sum_{a=1}^{\frac{N}{4}} d_{a}-\sum_{a=\left(\frac{N}{4}\right)+1}^{\frac{3 N}{4}} d_{a}+2 \sum_{a=(3 N / 4)+1}^{N} d_{a}\right) /(5 N / 2) \\
& w_{2}=\left(-\sum_{a=1}^{N / 4} d_{a}+\sum_{a=(3 N / 4)+1}^{N} d_{a}\right) / N
\end{aligned}
$$


$w_{3}=\left(-\sum_{a=(N / 4)+1}^{N / 2} d_{a}+\sum_{a=(N / 2)+1}^{3 N / 4} d_{a}\right) / N$

In general, the following model can be applied for various signal lengths:

$w_{2\left(\frac{N}{2}\right)}=w_{\left(\frac{N}{2}\right)}=\left(-\sum_{a=1}^{\frac{N}{N}} d_{a}+\sum_{a=N / N+1}^{2 N / N} d_{a}\right) /(N / N / 2)$

The new fashion of received PU signal, Y, contains only traffic with half of the length, $N_{\text {COMP }}$, of the original received PU signal using one level, as follows:

$$
\begin{aligned}
& \boldsymbol{Y}=\boldsymbol{\Phi}_{D D W T} \boldsymbol{X} \\
& N_{C O M P}=\frac{N}{2}
\end{aligned}
$$

The previous stage of spectrum compression reduces the consumed power in the front end up to $50 \%$. In addition, the compressed spectrum contains a big amount of traffic power with a little amount of noise power since the resultant spectrum conserves the signal measurements. Consequently, its detection performance gets better in low SNR.

On the other hand, using the CDCT algorithm as the second compression stage omits coefficients that have zero threshold rate. This stage can significantly reduce the noise variance and power consumption without signal resolution, specifications and measurements deterioration [23]. Moreover, it enhances the detection performance in lower SNR. Thus, applying the second compression stage on the compressed spectrum, which is a result of the first compression stage (DDWT), to obtain a big compression ratio with new length, $\mathrm{New}$, as follows:

$$
Z[k]=\left(\frac{(-1)^{k} Y[0]}{\sqrt{2}}+\sum_{k k=1}^{N_{C O M P}-1} Y[k k] \cos \left(\frac{\pi k(2 k k+1)}{2 N_{C O M P}}\right)\right) \quad \mathrm{k}=0,1, \ldots, \mathrm{K}-1
$$

As a matrix form, the next model compresses the stream by New:

$$
\boldsymbol{Z}=\boldsymbol{\Phi}_{C D C T} \boldsymbol{Y}
$$

Thus, the hybrid COMPSS scheme can be described as a structure, as shown in Figure 5. Finally, the test statistic for the final compressed spectrum results can be expressed to test the detection performance, as shown in the next equation.

$$
P S D_{\text {COMP }}=\frac{1}{N e w} \sum_{k=0}^{N e w-1}|Z[k]|^{2}
$$
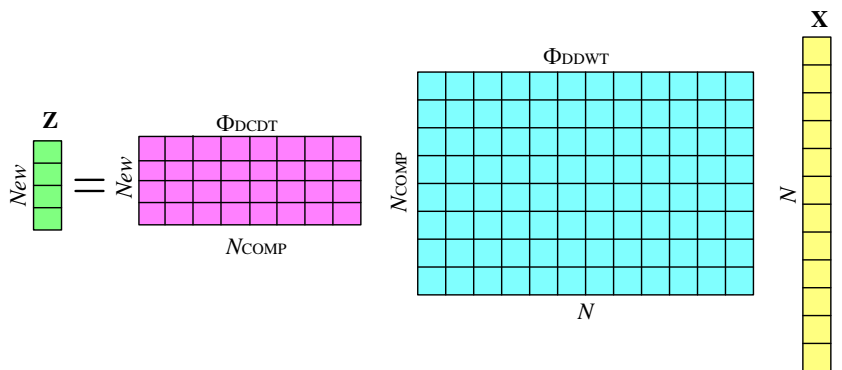

Figure 5. Hybrid COMPSS structure

By substituting $P S D_{\text {COMP }}$ and New into the CDCT for non-cooperative case and MCDCT for cooperative case algorithms, the cascaded systems can achieve a local decision for the first algorithm and a global decision for the second one as described below. However, the CDCT algorithm eliminates the coefficients that have a threshold value of zero or approaches to zero. After that, the algorithm obtains the PSD which has been compared with the predefined threshold to sense the PU signal and identify whether it is present or absent, as shown in Figure 7. 
As previously mentioned, each signal can be represented by a number of DCT coefficients to evaluate the received PU signal is first mathematically transformed to another domain using DCT-II family fusion:

$$
X[k]=\left(\frac{(-1)^{k} x[0]}{\sqrt{2}}+\sum_{n=1}^{N-1} x[n] \cos \left(\frac{\pi k(2 n+1)}{2 N}\right)\right) \sqrt{\frac{2}{N}} \quad k=0,1, \ldots, K-1
$$

After that, the new signal $Y[k]$ become shorter due to elimination of the zero threshold, can be modelled as follows:

$$
Y[k]=X[k] \quad k=0,1, \ldots, N e-1
$$

Next, the test statistic for active samples only is obtained. As shown in Figure 6, the threshold does not approach to or equals zero,

$$
P S D_{C D C T}=\frac{1}{N e} \sum_{k=0}^{N e-1}|Y[k]|^{2}
$$

where $P S D_{C D C T}$ denotes the test statistic and is lower in complexity than the raw periodogram, as investigated in the next section. Eliminating zero-valued coefficients also produces spectrum with its necessary data and a smaller number of samples. Therefore, the proposed ED technique has low mathematical and low computational complexity [24].

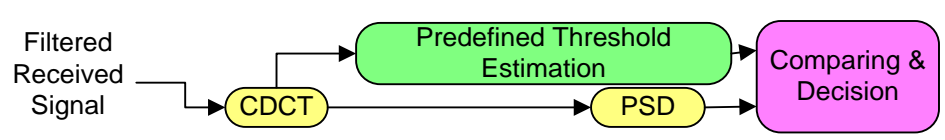

Figure 6. Proposed ED based on CDCT for SS block diagram

The comparing process can be modelled for the new stream, as follows:

$$
\begin{array}{ll}
P S D_{C D C T}[y]<\eta & H_{0} \\
P S D_{C D C T}[y] \geq \eta & H_{1}
\end{array}
$$

The main sensing aim is to maximise $P_{d}$ with minimised $P_{f}$ in a certain relationship. Such a relationship is called receiver operating characteristics (ROC), which describes the detection performance. In practice, to obtain the predefined threshold, $P_{f}$ is to be constant for a certain value, and this value should be small to prevent any harmful interference. The fixed values of $P_{f}$ and $P_{d}$ approaches are called CLFAR and constant local detection rate (CLDR), respectively. The approximated closed-form expressions for $P_{f}$ and $P_{d}$ under the AWGN channel are described in detail below, since $N \gg 250$. Suppose that $\gamma$ denotes the SNR of received PU signal, $X[k]$, and can be modelled as below:

$$
\gamma=\frac{\sigma_{s}^{2}}{\sigma_{w}^{2}}
$$

Thus, the probabilities of false alarm and detection can be formulated as follows:

$$
\begin{aligned}
& P_{f}=Q\left(\frac{\eta / \sigma_{w}^{2}-1}{\sqrt{\left(\frac{2}{N}\right)}}\right) \\
& P_{d}=Q\left(\frac{\eta / \sigma_{w}^{2}-(\gamma+1)}{\sqrt{\left(\frac{2}{N e}\right)}(\gamma+1)}\right)
\end{aligned}
$$

To switch the SNR of the original received PU signal, $X[k]$, to the SNR of new-length PU signal, $Y[k]$, the following ratio can be derived according to the lengths of both signals, where $\alpha$ represents the length ratio: 
$\alpha=\frac{N}{N e}$

After that, this ratio is multiplied by the SNR of the originally received PU signal to obtain the SNR of the new-length PU signal. Thus, (21) will become:

$$
P_{d}=Q\left(\frac{\eta / \sigma_{w}^{2}-\alpha(\gamma+1)}{\sqrt{\left(\frac{2}{N e}\right) \alpha(\gamma+1)}}\right)
$$

From the definition of $P_{f}$ and $P_{m d}$, a probability of error, $P_{e}$, can be considered, as follows [18]:

$P_{e}=P_{f}+P_{m d}=1+P_{f}-P_{d}$

Furthermore, $\eta$ should be estimated according to the $P_{f}$ rate and then the noise variance is required to obtain $\eta$ [25]. Accordingly, $\eta$ can be derived from (20), as follows:

$$
\eta=\sigma_{w}^{2}\left(1+\sqrt{\left(\frac{2}{N}\right)} Q^{-1}\left(P_{f a}\right)\right)
$$

To analyse the noise variance effect, the predefined threshold can be normalised with variance of noise, as shown below:

$$
\eta /_{\sigma_{w}^{2}}=\left(1+\sqrt{\left(\frac{2}{N}\right)} Q^{-1}\left(P_{f a}\right)\right)
$$

To perform it, the previous optimal rates must be used in (20 and 23) to obtain the crucial factors $\mathrm{Ne}$ and $\eta$. In this research, the predefined threshold can be obtained from (42) using CLFAR, as shown below:

$$
\eta=\sigma_{w}^{2}\left(1+\sqrt{\left(\frac{2}{N}\right)} Q^{-1}(0.01)\right)
$$

Substitute the predefined threshold into (23) to figure out the best length for the received PU signal using CLDR:

$$
N e=\left(\frac{\alpha(\gamma+1) Q^{-1}\left(P_{d}\right)}{\sqrt{2}\left(\eta / \sigma_{w}^{2}-\alpha(\gamma+1)\right)}\right)^{2}
$$

Although the cancelled coefficients do not affect signal detection purposes, obtaining the correlation between the original length PU signal and the new-length PU signal can realize the sensing improvement. The linear cross-correlation model can be stated as follows:

$$
Z_{X Y}=X[k] \otimes Y[k] \quad k=0,1, \ldots, N+N e-1
$$

where $\otimes$ denotes linear cross-correlation operation.

As aforementioned, each SU sends its local decision to the fusion center (FC) which decides a global decision. However, the MCDCT algorithm eliminates the coefficients that have a threshold value of zero or approaches to zero according to the number of SUs. After that, the algorithm obtains the global decision via OR-rule, and sends its decision to all SUs, as shown in Figure 7.

The FC must decide the previous desire values from (20) and (23) each user should make a local decision regarding the global one. Therefore, the local decision can be formulated by using a CGFAR and a constant global detection rate (CGDR) from (20) and (23) as follows:

$$
\begin{aligned}
& P_{f c s s}=1-\left(1-Q_{f}\right)^{\frac{1}{n s u}}=1-(0.99)^{\frac{1}{n s u}} \\
& P_{d c s s}=1-\left(1-Q_{d}\right)^{\frac{1}{n s u}}=1-(0.1)^{\frac{1}{n s u}}
\end{aligned}
$$




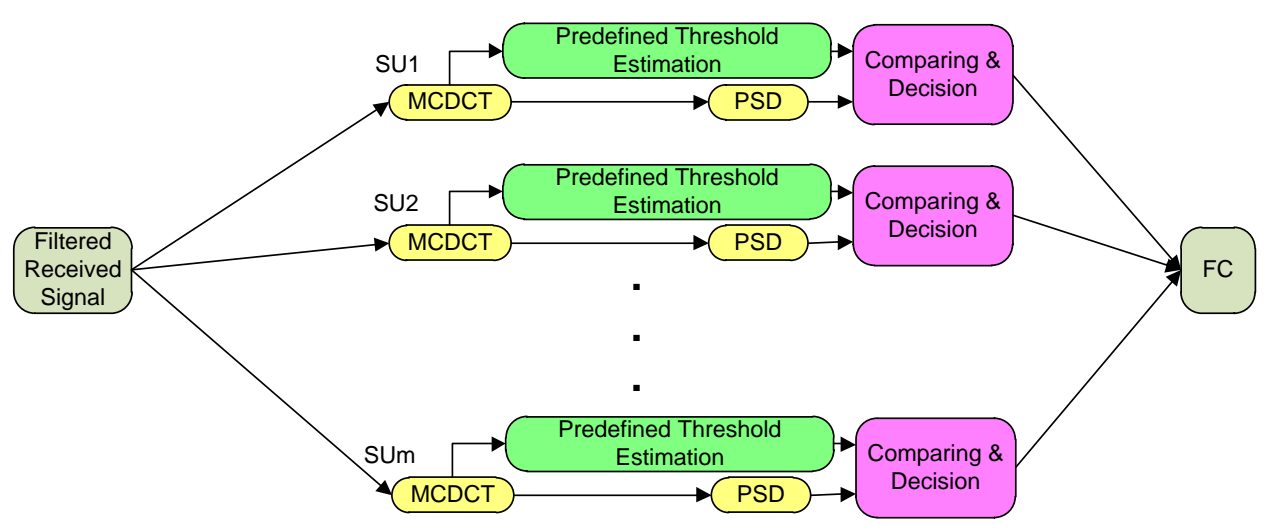

Figure 7. Proposed ED based on MCDCT for SS block diagram

Substitute $P_{f c s s}$ and $P_{d c s s}$ in (27) and (28), respectively to find the crucial factors $N e$ and $\eta$ and then design the cooperative case detector as shown below:

$$
\begin{aligned}
& \eta=\sigma_{w}^{2}\left(1+\sqrt{\left(\frac{2}{N}\right)} Q^{-1}\left(1-(0.99)^{\frac{1}{n s u}}\right)\right) \\
& N e=\left(\frac{(\alpha \gamma+1) Q^{-1}\left(1-(0.1)^{\frac{1}{n s u}}\right)}{\sqrt{2}\left(\eta^{\eta} / \sigma_{w}^{2}-(\alpha \gamma+1)\right)}\right)^{2}
\end{aligned}
$$

Thus, each user in a centralized cooperative CRN should decide locally from (30), (31) to help the FC to decide on desired global ratios.

\section{RESULTS AND DISCUSSION}

In this section, a system-level simulation was built by using MATLAB to achieve the specifications of CRN standard [11]. The simulation environment considered an up-link transmission from PU equipment, which is located in some region. This region contained about 100 SU devices and was covered by an AWGN channel for various SNR values from zero till $-50 \mathrm{~dB}$ and other simulation parameters as shown in Table 1.

Table 1. The simulation parameters

\begin{tabular}{ll}
\hline Up-link Parameters & Value \\
\hline Transmission mode & 2 K mode \\
Number of FFT & 2048 samples \\
Bandwidth & 6,7, and $8 \mathrm{MHz}$ \\
Sampling time & $7 / 48,7 / 56$, and $7 / 64$ \\
Cyclic prefix & $1 / 4,1 / 8,1 / 16$, and $1 / 32$ \\
Number of subcarriers & 1705 samples \\
Modulation scenarios & QPSK and 16-QAM \\
Channel type & AWGN channel \\
SNR & $-50-0$ dB \\
\hline
\end{tabular}

\subsection{Detection performance for fixed $Q_{f}$}

Figure 8 exhibits a surface plot for the the global probability of detection versus the number of SUs and SNR, whereas the global probability of false alarm is constant at 0.01 and one SU only. From Figure 8, it can be deduced that the performance is excellent for every SNR and SU number that is equal to and greater than 20 users. The values of the original stream, first compression stage, and second compression stage lengths are listed in Table 2, where $\epsilon_{h}$, and $\epsilon_{l}$ denote the higher and lower removed power ratios, respectively. From Table 2, the resultant compression ratios are around $80 \%, 80.5 \%, 81.5 \%$, and $81 \%$ for the 16-QAM $(\mathrm{G}=4)$, QPSK $(\mathrm{G}=4)$, 16-QAM $(\mathrm{G}=32)$, and QPSK $(\mathrm{G}=32)$, scenarios respectively. This figure also shows that the noise variance is significantly reduced in both stages, especially for high numbers of SUs in the CRN. 


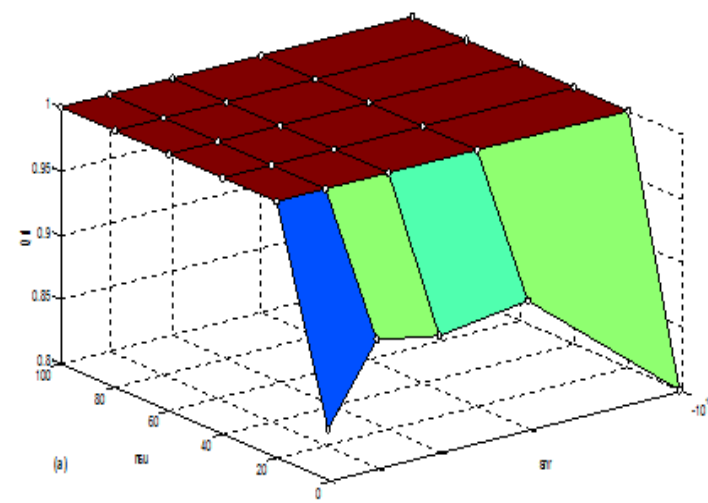

(a)

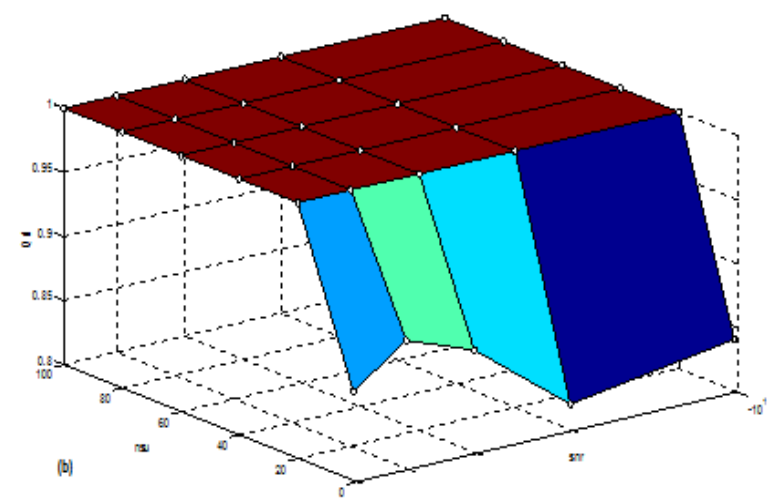

(b)

Figure 8. $Q_{d}$ of DDWT-CDCT, $Q_{f}=0.01, n s u=1-100$ users and SNR=-50-0 dB,

(a) $\mathrm{G}=1 / 4$ for $16-\mathrm{QAM}$ scenario, (b) $\mathrm{G}=1 / 4$ for $\mathrm{QPSK}$ scenario

Table 2. $Q_{d}, \epsilon_{h}$, and $\epsilon_{l}$ of DDWT-CDCT for $Q_{f}=0.01$, and different values of cyclic prefix

\begin{tabular}{cccccccc}
\hline \multicolumn{2}{c}{$16-\mathrm{QAM}(\mathrm{G}=4)$} & \multicolumn{2}{c}{ QPSK $(\mathrm{G}=4)$} & \multicolumn{2}{c}{$16-\mathrm{QAM}(\mathrm{G}=32)$} & $\mathrm{QPSK}(\mathrm{G}=32)$ \\
\hline$N=2560$ & $\epsilon_{l}=-55.23 \mathrm{~dB}$ & $N=5120$ & $\epsilon_{l}=-63 \mathrm{~dB}$ & $N=2112$ & $\epsilon_{l}=-53 \mathrm{~dB}$ & $N=4224$ & $\epsilon_{l}=-60 \mathrm{~dB}$ \\
$N_{\mathrm{COMP}}=1280$ & $N e w=1117$ & $N_{\mathrm{COMP}}=2560$ & $N e w=2318$ & $N_{\mathrm{COMP}}=1056$ & $N e w=907$ & $N_{\mathrm{COMP}}=2112$ & $N e w=1879$ \\
$N e w / \epsilon_{h}=516$ & $\epsilon_{h}=-20 \mathrm{~dB}$ & $N e w / \epsilon_{h}=998$ & $\epsilon_{h}=-20 \mathrm{~dB}$ & $N e w / \epsilon_{h}=389$ & $\epsilon_{h}=-20 \mathrm{~dB}$ & $N e w / \epsilon_{h}=804$ & $\epsilon_{h}=-20 \mathrm{~dB}$ \\
\hline
\end{tabular}

\subsection{Detection performance for fixed SNR}

The surface plot of Figure 9 shows the ROC versus the number of SUs where the SNR is constant at $-50 \mathrm{~dB}$. The signal is watched by a number of SUs that varies between 1 and 100 users along the ROC. From Figure 9, it can be seen that the global detection probability is excellent for 20 users or more for all cyclic prefix and scenarios. From one user till 20 users in the 16-QAM scenario, the global probability of false alarm changes from zero to 0.07 and zero to 0.03 , and the resultant global detection probability is from 0.9 to 1 . In terms of the same range of users in the QPSK scenario, the global false alarm probability varies between zero to 0.07 and zero to 0.1 , and the resultant global detection probability is from 0.9 to 1 .

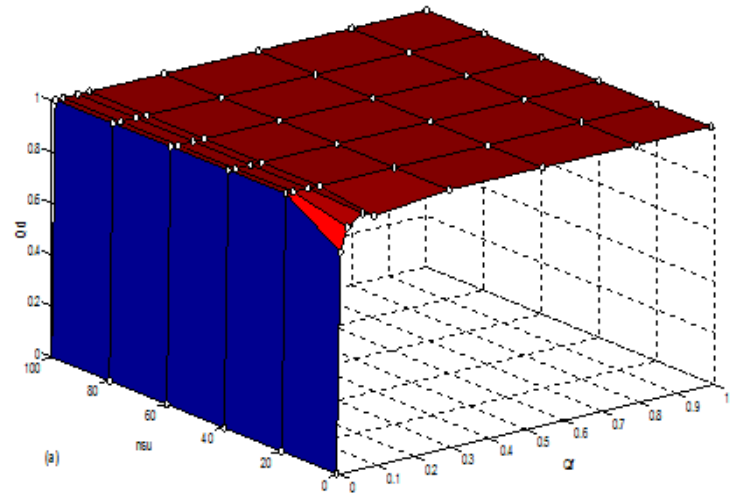

(a)

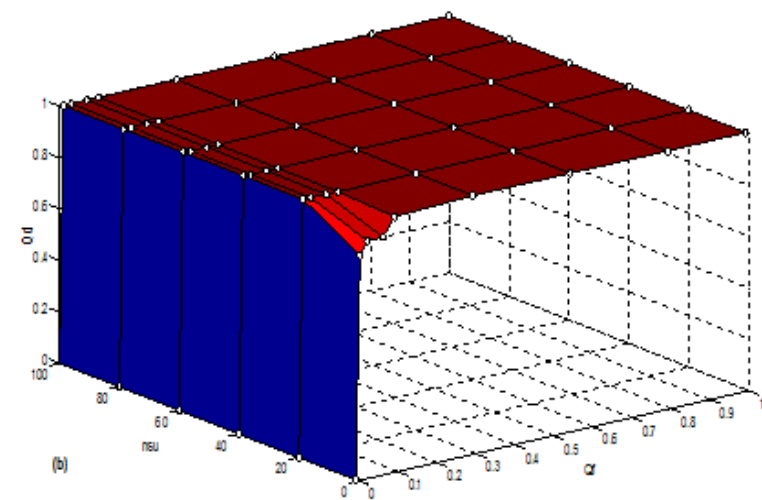

(b)

Figure 9. $Q_{d}$ of DDWT-CDCT, SNR=-50 dB, $n s u=1-100$ users and $Q_{f=0}-1$,

(a) $\mathrm{G}=1 / 4$ for $16-\mathrm{QAM}$ scenario, (b) $\mathrm{G}=1 / 4$ for $\mathrm{QPSK}$ scenario

From the Table 3, it can be seen that the first stage achieved compression ratios that are the same as those of fixed global false alarm probability. From these values of lengths, the overall compression ratios are $80 \%, 80 \%, 81 \%$, and $81 \%$ for $16-\mathrm{QAM}(\mathrm{G}=4)$, QPSK $(\mathrm{G}=4), 16-\mathrm{QAM}(\mathrm{G}=32)$, and QPSK $(\mathrm{G}=32)$, scenarios respectively. The difference in ratios between the case of fixed global false alarm probability and the fixed SNR exhibits the effect of the SNR wall for different scenarios and cyclic prefix ratios. 
Table 3. $Q_{d}, \epsilon_{h}$, and $\epsilon_{l}$ of DDWT-CDCT for SNR=-50 dB, and different values of cyclic prefix

\begin{tabular}{cccccccc}
\hline \multicolumn{2}{c}{ 16-QAM $(\mathrm{G}=4)$} & \multicolumn{2}{c}{ QPSK $(\mathrm{G}=4)$} & \multicolumn{2}{c}{ 16-QAM $(\mathrm{G}=32)$} & \multicolumn{2}{c}{ QPSK $(\mathrm{G}=32)$} \\
\hline$N=2560$ & $\epsilon_{l}=-55.23 \mathrm{~dB}$ & $N=5120$ & $\epsilon_{l}=-63 \mathrm{~dB}$ & $N=2560$ & $\epsilon_{l}=-55.23 \mathrm{~dB}$ & $N=5120$ & $\epsilon_{l}=-63 \mathrm{~dB}$ \\
$N_{\text {COMP }}=1280$ & New $=1109$ & $N_{\text {COMP }}=2560$ & New $=2324$ & $N_{\text {COMP }}=1280$ & New $=1109$ & $N_{\text {COMP }}=2560$ & New $=2324$ \\
New $/ \epsilon_{h}=517$ & $\epsilon_{h}=-20 \mathrm{~dB}$ & $N e w / \epsilon_{h}=1002$ & $\epsilon_{h}=-20 \mathrm{~dB}$ & New $/ \epsilon_{h}=517$ & $\epsilon_{h}=-20 \mathrm{~dB}$ & New $/ \epsilon_{h}=1002$ & $\epsilon_{h}=-20 \mathrm{~dB}$ \\
\hline
\end{tabular}

\subsection{Discussion}

All in all, the proposed Hybrid COMPSS scheme decreased the consumed power by a ratio up to $80 \%$ regarding signal measurements. Thus, the pollution of radii that emits from equipment will significantly decrease. Furthermore, the first stage operations are too simple and are equal to $2 \mathrm{~N}$ operations only, which is the average of additions and subtractions. The second stage increased the compression ratio, enhanced the reliability and decreased the variance of noise and error. Moreover, both stages used smaller signals and then required a short time to sense although there are two stages and not only one. Table 4 summarizes the different parameters among the Hybrid COMPSS, the stationary wavelet edge transform [3] and signal matrix estimation [10] algorithms.

Table 4. $Q_{d}, \epsilon_{h}$, and $\epsilon_{l}$ of DDWT-CDCT for SNR=-50 dB, and different values of cyclic prefix

\begin{tabular}{lllll}
\hline Parameters & $\begin{array}{l}\text { Stationary Wavelet Edge } \\
\text { Transform [3] Algorithm }\end{array}$ & $\begin{array}{l}\text { Signal Matrix Estimation } \\
{[10] \text { Algorithm }}\end{array}$ & $\begin{array}{l}\text { Hybrid } \\
\text { COMPSS }\end{array}$ & Comparison \\
\hline $\begin{array}{l}\text { Detection Performance for } \\
\text { non-cooperative }\left(P_{d}, P_{f a}\right)\end{array}$ & $(0.8,0.09)$ & N/A & $(0.9,0.01)$ & $\begin{array}{l}\text { Hybrid COMPSS scheme } \\
\text { is better for one SU }\end{array}$ \\
$\begin{array}{l}\text { Detection Performance for } \\
\text { cooperative }\left(Q_{d}, Q_{f a}\right)\end{array}$ & N/A & $(0.67,0.01)$ & $(0.99,0.01)$ & $\begin{array}{l}\text { Hybrid COMPSS scheme } \\
\text { is better for Multi-SUs }\end{array}$ \\
$\begin{array}{l}\text { Compression Ratio } \\
\text { SNR Wall/dB }\end{array}$ & $50 \%$ & $70 \%$ & $81.5 \%$ & $\begin{array}{l}\text { Higher Compression Ratio } \\
\text { SNR Wall lower 5 times }\end{array}$ \\
SU Number & -10 & 1 & -50 & $\begin{array}{l}\text { Used for non-cooperative } \\
\text { and cooperative users }\end{array}$ \\
\hline
\end{tabular}

\section{CONCLUSION}

In this paper, a hybrid COMPSS schemes for non-cooperative and cooperative SUs are derived. These schemes were constructed using two stages; DDWT and CDCT/MCDCT for one scheme. The first stage enhanced the detection performance, achieved 50\% compression ratio that shortened the sensing period, decreased the noise variance, and had low cost by reducing computational complexity. On the other hand, the second stage enhanced the detection performance furthermore to achieve up to $30 \%$ compression ratio ( $80 \%$ overall compression ratio), reduced the SNR wall, and had too low cost since it dealt with half of the original signal. These achievements were achieved for both modulation scenarios and for cooperative and non-cooperative CRNs. Furthermore, a reconstruction of the signal was not required since both hybrid COMPSS schemes kept the necessary measurements of the detected signal.

\section{REFERENCES}

[1] I. F. Akyildiz, et al., "NeXt generation/dynamic spectrum access/cognitive radio wireless networks: A survey," Computer networks, vol. 50, no. 13, pp. 2127-2159, 2006.

[2] Q. Zhao, et al., "Energy efficiency of compressed spectrum sensing in wideband cognitive radio networks," EURASIP Journal on Wireless Communications and Networking, vol. 2016, no. 1, pp. 83-93, 2016.

[3] S. E. El-Khamy, et al., "A stationary wavelet transform approach to compressed spectrum sensing in cognitive radio," International Journal of Communication Systems, vol. 30, no. 7, p. e3140, 2016.

[4] S. D. Borde and K. R. Joshi, "Enhanced signal detection slgorithm using trained neural network for cognitive radio receiver," International Journal of Electrical and Computer Engineering (IJECE), vol. 9, no. 1, pp. 323-331, 2019.

[5] P. Varade, et al., "Throughput Maximization of Cognitive Radio Multi Relay Network with Interference Management," International Journal of Electrical \& Computer Engineering (IJECE), vol. 8, no. 4, pp. 2230-2238, 2018.

[6] P. M. Nam, et al., "Performance of cluster-based cognitive multihop networks under joint impact of hardware noises and non-identical primary co-channel interference," TELKOMNIKA Telecommunication Computing Electronics and Control, vol. 17, no. 1, pp. 49-59, 2019.

[7] E. Astaiza, et al., "Compressive local wideband spectrum sensing algorithm for multiantenna cognitive radios," in 2016 8th IEEE Latin-American Conference on Communications (LATINCOM), pp. 1-6, 2016.

[8] W. Wang, et al., "Fast compressed spectrum sensing on positioning technology," in 2016 IEEE International Conference on Ubiquitous Wireless Broadband (ICUWB), pp. 1-4, 2016.

[9] F. Li, et al., "A Novel Approach to Wideband Spectrum Compressive Sensing Based on DST for Frequency Availability in LEO Mobile Satellite Systems," Mathematical Problems in Engineering, pp. 1-13, 2016. 
[10] S. Ren, et al., "A low complexity sensing algorithm for wideband sparse spectra," IEEE Communications Letters, vol. 21, no. 1, pp. 92-95, 2016.

[11] L. Xu, et al., "DRiVE-ing to the Internet: Dynamic radio for IP services in vehicular environments," in Proceedings 25th Annual IEEE Conference on Local Computer Networks. LCN 2000, pp. 281-289, 2000.

[12] D. Pineda and C. Hernandez, "Cognitive radio for TVWS usage," TELKOMNIKA Telecommuincation Computing Electronics and Control, vol. 17, no. 6, pp. 2735-2746, 2019.

[13] S. Razmi and N. Parhizgar, "Adaptive resources assignment in OFDM-based cognitive radio systems," International Journal of Electrical and Computer Engineering, vol. 9, no. 3, pp. 1935-1943, 2019.

[14] D. T. Do, et al., "Cooperative underlay cognitive radio assisted NOMA: secondary network improvement and outage performance," TELKOMNIKA Telecommunication Computing Electronics and Control, vol. 17, no. 5, pp. 2147-2154, 2019.

[15] E. Candes and J. Romberg, "Sparsity and incoherence in compressive sampling," Inverse problems, vol. 23, no. 3, p. 969, 2007.

[16] E. J. Candès and M. B. Wakin, "An introduction to compressive sampling [a sensing/sampling paradigm that goes against the common knowledge in data acquisition]," IEEE signal processing magazine, vol. 25, no. 2, pp. 21-30, 2008.

[17] F. Salahdine, et al., "A survey on compressive sensing techniques for cognitive radio networks," Physical Communication, vol. 20, pp. 61-73, 2016.

[18] D. L. Donoho, "Compressed sensing," IEEE Transactions on information theory, vol. 52, no. 4, pp. 1289-1306, 2006.

[19] N. Umezu, et al., "2D wavelet transform data compression with error level guarantee for Z-map models," Journal of Computational Design and Engineering, vol. 4, no. 3, pp. 238-247, 2017.

[20] P. Saravanan, et al., "Digital image watermarking using daubechies wavelets," in 2016 3rd International Conference on Signal Processing and Integrated Networks (SPIN), pp. 57-62, 2016.

[21] Y. Zheng, et al., "An advanced image fusion algorithm based on wavelet transform: incorporation with PCA and morphological processing," in Image processing: algorithms and systems III, International Society for Optics and Photonics, vol. 5298, pp. 177-187, 2004.

[22] I. Daubechies, "Ten lectures on wavelets," CBMS-NSF regional Conference Series in Applied Mathematics, vol. 61, 1992.

[23] S. A. Golestaneh and D. M. Chandler, "No-reference quality assessment of JPEG images via a quality relevance map," IEEE Signal Processing Letters, vol. 21, no. 2, pp. 155-158, 2013.

[24] N. R. Banavathu and M. Z. A. Khan, "Optimal n-out-of-k voting rule for cooperative spectrum sensing with energy detector over erroneous control channel," in 2015 IEEE 81st Vehicular Technology Conference (VTC Spring), pp. 1-5, 2015.

[25] T. Yucek and H. Arslan, "A survey of spectrum sensing algorithms for cognitive radio applications," IEEE communications surveys \& tutorials, vol. 11, no. 1, pp. 116-130, 2009.

\section{BIOGRAPHIES OF AUTHORS}

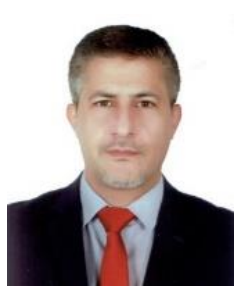

Montadar Abas Taher. He received the B.Sc. degree in Electronics and Communication Engineering and the M.Sc. degree in Satellite Engineering from Al-Nahrain University, Iraq, in 2000 and 2003, respectively, and the Ph.D. degree from the National University of Malaysia, in 2015. $\mathrm{He}$ was with Motorola as a Communications Engineer, from 2005 to 2009. Since 2015, he is the Head of the Communications Engineering Department, University of Diyala. His main research interests include OFDM, CDMA, MC-CDMA, 4G, and 5G. He is a Reviewer for some respected international journals.

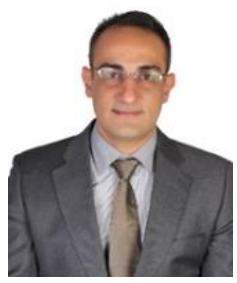

Mohammad Z. Ahmed was Born in Amman (Jordan) in 1980. He received his Ph.D. in Micro engineering and nano electronics in 2015 from The National University of Malaysia. In 2007 he received his M.Sc. in electronics system design from the University of Science Malaysia. In 2003, he received his B.Sc. in electrical engineering from Baghdad University. In 2016 he joined Al-Hussein Bin Talal University as a lecturer.

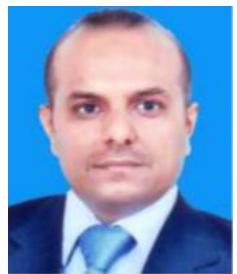

Emad Hmood Salman received the B.Sc. degree in Electrical Engineering from University of Baghdad, Iraq, 2003. Then, he received the M.Sc. in Electrical Engineering/Electronics and Communications Engineering from same university, in 2007. Since 2007, he is working as a lecturer in College of Engineering, University of Diyala, Iraq. He received the Ph.D. in Wireless Communications Engineering field/Computer and Communication Systems Engineering Department at Universiti Putra Malaysia, in 2019. His main research interests are Cognitive Radio, (3-6) G standards. 